\title{
Filigrane
}

Écoutes psychanalytiques

\section{Transmettre la psychanalyse : l'exemple du Columbia Parent-Infant Program à New York}

\section{Christine Anzieu-Premmereur}

Volume 27, numéro 1, 2018

La transmission de la psychanalyse

URI : https://id.erudit.org/iderudit/1055601ar

DOI : https://doi.org/10.7202/1055601ar

Aller au sommaire du numéro

Éditeur(s)

Revue Santé mentale au Québec

ISSN

1192-1412 (imprimé)

1911-4656 (numérique)

Découvrir la revue

Citer cet article

Anzieu-Premmereur, C. (2018). Transmettre la psychanalyse : l'exemple du Columbia Parent-Infant Program à New York. Filigrane, 27(1), 31-43.

https://doi.org/10.7202/1055601ar
Résumé de l'article

Comment transmettre les fondements de la psychanalyse dans le cadre de la formation à la psychothérapie parents-nourrissons ? Comment peut-on appliquer la métapsychologie freudienne au travail analytique avec des nourrissons et leurs parents ? Des concepts tels que défense, transfert, sexualité infantile, Moi corporel et surmoi peuvent-ils s'appliquer dès le début de la vie ? L'importance de la relation libidinale, associée à la notion de défense contre l'angoisse primitive et au rôle des projections maternelles inconscientes, sera illustrée par des vignettes cliniques. 


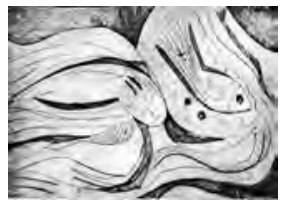

\title{
Transmettre la psychanalyse: I'exemple du Columbia Parent-Infant Program à New York
}

\author{
Christine Anzieu-Premmereur
}

\begin{abstract}
Résumé: Comment transmettre les fondements de la psychanalyse dans le cadre de la formation à la psychothérapie parents-nourrissons? Comment peut-on appliquer la métapsychologie freudienne au travail analytique avec des nourrissons et leurs parents? Des concepts tels que défense, transfert, sexualité infantile, Moi corporel et surmoi peuvent-ils s'appliquer dès le début de la vie? L’importance de la relation libidinale, associée à la notion de défense contre l'angoisse primitive et au rôle des projections maternelles inconscientes, sera illustrée par des vignettes cliniques.
\end{abstract}

Mots clés: thérapie parent-bébé; transmission de la psychanalyse; plaisir libidinal; théorie des pulsions; destructivité; fonction maternelle.

\begin{abstract}
How can we operate the transmission of psychoanalytic thinking while training students and professionals to parent-infant psychotherapy? How can some classical Freudian concepts be applied to help the analyst working with dyads, such as defense, transference, infantile sexuality, the role of body ego and super ego in the first year of life? The importance of libidinal pleasure in relation with the mother, the notion of defense towards primitive anxiety and the role of the unconscious maternal functioning are helpful to help the child as well as the parents to regulate their interactions.
\end{abstract}

Key Words: parent-infant therapy; transmission of psychoanalysis; libidinal pleasure; drive theory; destructiveness; maternal function.

'est un honneur et un plaisir pour moi d'être à Montréal, si proche de la psychanalyse française, mais avec une identité forte. André Lussier en est le meilleur exemple.

La transmission a plusieurs vecteurs; elle peut être individuelle ou institutionnelle, verticale ou horizontale. L'héritage est là, mais est-il intégrable s'il n'y a pas de refus ou de déni? Comment le revendiquer dans un monde pour lequel la position des générations s'est modifiée? Les parents cherchent à transmettre à leurs enfants, mais ce n'est pas toujours ce qu'ils souhaitent 
qui passe d'une génération à l'autre: ce sont aussi des fantômes, du négatif, des secrets.

Un jeu ambivalent d'identification et de refus fait de l'héritage une matière confuse qui doit être appropriée activement. Dans le domaine de la psychanalyse, les fonctions de cadre et de Surmoi donnent une forme à la pensée. Reste pourtant à ouvrir un espace pour la nouveauté, parfois critique. Les modèles personnels sont pour ce faire essentiels, modèles de créativité ou de soumission au passé, de liberté d'innover et de remise en question, ou encore de répétition à l'identique.

Les débats entre les tenants d'Anna Freud et ceux de Mélanie Klein, la position polémique de Winnicott par rapport à Freud comme avec M. Klein, les remises en question de la lecture de Freud et de la formation analytique par Lacan, le bouleversement introduit par Bion, et d'autres plus récents, montrent combien l'histoire des écoles analytiques et les enjeux institutionnels et de pouvoir ont façonné les positions personnelles des nouveaux élèves en psychanalyse.

Comme on a pu le voir dans les instituts, les phénomènes d'imitation des maitres et le conservatisme dogmatique d'une théorie empêchent de penser et d'innover.

Les affects jouent un rôle essentiel dans la capacité d'absorber l'héritage à travers une lecture critique: le plaisir d'apprendre, l'idéalisation des enseignants, l'ambition de devenir capable de penser l'inconscient et la sexualité, l'angoisse de ne pas en avoir le droit, la complicité avec le groupe des pairs, la rigueur instaurée par le cadre de la formation, les comportements professionnels des collègues, et surtout le modèle de ce que son propre analyste fait, tout ceci façonne un mode complexe d'envie et de rejet.

Les enfants de psychanalystes ne deviennent pas tous analystes, loin de là. Ceux qui peuvent en franchir les obstacles narcissiques et œedipiens tentent le coup. Ne plus être «l'enfant de» et ainsi regrouper son identité relève de l'analyse personnelle, mais l'essentiel est de trouver un champ de pensée et de travail original et personnel.

La traversée de l'Atlantique a été pour moi une grande chance. Ce n'était pas mon choix, mais celui de mon mari, chercheur en immunologie. Le choc culturel, la douleur de la séparation, le casse-tête d'avoir à gérer deux enfants adolescents en révolte, tous ces problèmes classiques de l'immigration non tragique m'ont permis d'accumuler une expérience et un savoir nouveaux qui ont rouvert mon auto-analyse. Connaitre l'histoire du continent américain, sa culture, comment la psychanalyse y a été transformée, la complexité 
des histoires d'immigration, la découverte de la langue anglaise, précise et pragmatique, ont formé mon esprit à s'assouplir pour s'adapter. Un travail personnel sur mes origines, mon identité, mes idéalisations et mes erreurs a porté ses fruits après quelques souffrances.

Je me suis mise au rang des règles des instituts new-yorkais après bien des déboires, et je rends hommage à Hélène Tessier pour son livre sur la psychanalyse américaine qui m'a ouvert les yeux.

L'épreuve s'est transformée en chance, la population francophone étant immense dans la région de New York. Devenue «la psychanalyste de la Société psychanalytique de Paris à New York» grâce aux collègues qui me rappelaient souvent que j'avais une position unique, j'ai été accueillie à la société de Columbia en mal de cliniciens travaillant avec les tout-petits. J'y ai découvert que mon expérience passée avait une valeur spécifique. Ce qui me paraissait un acquis évident devenait une nouveauté intéressante.

Après quelques aventures avec la censure - tout texte kleinien étant banni de mon curriculum pendant 3 ans -, j'ai pu maintenir mes positions théoriques et les défendre, et enfin développer une formation à la psychothérapie psychanalytique parent-nourrisson. L'échange avec des psychologues américains, jeunes et prompts à discuter toute idée nouvelle, et la présence de nombreux étudiants internationaux venus de cultures différentes m’ont remuée et stimulée, m’obligeant à revoir mes théories et à réviser des préjugés faciles à maintenir en France, mais pas ailleurs.

\section{Transmettre}

Je ne donnais pas à l'origine des formations à la psychanalyse; ce fut un choix dû au changement de pays. J'ai préféré consacrer mon énergie à m'adapter à la nouveauté et à cette formidable opportunité de créer un département autour d'enfants de moins de 3 ans. Cette formation s'adresse à de jeunes médecins et psychologues, qui découvrent la pensée analytique et les modèles théoriques qui s'appliquent à la compréhension du monde interne du bébé et de la dynamique interne des parents, ainsi que les techniques d'intervention. Une moitié de chaque groupe d'une dizaine d'étudiants se dirige ensuite vers une formation analytique. Il leur est demandé de commencer une analyse pendant la formation. La plupart continuent, après les deux années de formation, à être en supervision pour les cas parentsenfants. Comme je fais partie du comité sur la psychanalyse d'enfant de l'Association psychanalytique internationale, je valide l'intégration du cursus en analyse d'enfant avec la formation analytique adulte. 
Transmettre est un système interactif, qui fonctionne aussi du plus jeune vers l'enseignant. Cela implique de revisiter les théories, les relire sous un jour contemporain, répondre au questionnement des élèves, qui ont d'autres repères et d'autres curiosités, ou des expériences cliniques et médicales nouvelles. De même que l'expérience réelle avec un bébé qui vient d'arriver dans le monde nous confronte à l'imprévisible, à nos émotions, à la vulnérabilité redécouverte, à la joie narcissique, tout ce qui permet à de nouveaux parents de se faire parents, de même le petit qui se transforme au cours d'une thérapie nous en apprend toujours plus sur l'appareil psychique, le sien comme le nôtre, si on accepte de l'observer "sans mémoire ni désir». Mettre les étudiants en situation de vivre cette expérience par l'observation du nourrisson et la supervision de groupe qui s'ensuit, c'est leur transmettre la capacité d'auto observation et d'auto analyse, tout en se remettant soi-même en situation de découverte de nouvelles émotions et de pensées jusque-là refoulées ou non élaborées.

La transmission se fait aussi entre pairs. La dynamique du groupe est un élément essentiel pour laisser des échanges actifs dans la diversité. C'est parfois un grand plaisir d'enseigner à un groupe dynamique qui se réjouit de découvrir la psychanalyse tout en l'interrogeant.

Dans le contexte américain, les discussions portent beaucoup plus sur l'efficacité des traitements que sur leur validité métapsychologique.

Il existe depuis longtemps maintenant un consensus pour reconnaître que les patients se présentent plus souvent avec des symptômes autres que ceux des névroses dites «classiques». Il y a, d'une part, une prégnance du questionnement narcissique, voire des pathologies du narcissisme, avec une difficulté à la subjectivation, des modes d'expression privilégiant l'acte et le corps et, d'autre part, une vulnérabilité extrême quant à l'estime de soi, et au regard porté par autrui. Ce sont des situations qui nous portent aux limites de l'analyse et réinterrogent nos acquis métapsychologiques. Le symptôme se réfugie de plus en plus souvent dans l'actuel ou le corporel, voire le comportement (conduites addictives, violence.) Devant ces situations, nous sommes amenés à reconsidérer, pour nombre de cas, les modalités du traitement analytique, à approfondir la réflexion théorico-clinique et à penser une psychothérapie psychanalytique qui a pour appui essentiel le travail du transfert et du contre-transfert.

Les élèves sont incités à se garder d'une relation en miroir qui ferait disparaître la dimension asymétrique de la relation. La rigueur du cadre passe par la régularité des séances, leur durée, la totale liberté de parole. Pour des 
jeunes adultes sans expérience de l'analyse, c'est une découverte qui entraine de la résistance.

Le cadre est maintenu par la personne de l'analyste; il est la surface de travail et le contenant. La présence physique, corporelle, associe aussitôt les manques et défaillances de l'objet primaire. L'analyste y est sensible, mais se centre sur les manifestations du contre-transfert, sans viser à remplacer l'objet du passé. La personne de l'analyste a souvent un rôle de gardien de la vie psychique, une fonction antidépressive. Mais le face-à-face comporte aussi des risques. Le risque de faire de la pédagogie, l'illusion de tout comprendre, le risque de séduction et de connivence. On connaît les fausses adhésions des patients aux propos de l'analyste, le renforcement des défenses, les déplacements de culpabilité aux propos de déculpabilisation.

Les analystes de la côte Est ont tendance à privilégier le maintien du holding au premier plan, et ce au prix de la disparition des pulsions, des fantasmes inconscients, des événements infantiles. On ne réfère plus à la sexualité infantile.

Apprendre aux nouveaux venus que le psychanalyste ne porte pas son intérêt sur le symptôme et que le soulagement d'un symptôme n'a souvent pour effet que son déplacement et empêche de rester centré sur le fonctionnement psychique est devenu très difficile.

Je tente de montrer aux étudiants ce que Raymond Cahn écrivait il y a une dizaine d'années: le psychanalyste s'attache plutôt aux capacités de changement intrapsychique et aux nouveaux rapports que l'analysant établit avec ses désirs, ses conflits, ses amours et ses haines, son image de luimême, ses idéaux, ses sentiments de culpabilité, ses aménagements défensifs et sa relation aux objets externes et à la réalité (Cahn, 2002). Pour se dire convaincu «de la profonde unité de l'écoute et de l'action psychanalytiques quelles que soient l'organisation de la psyché et les circonstances de la rencontre», il s'agit de remettre l'héritage continuellement en question tout en le respectant, de le développer sans se cloisonner, mais aussi de préserver sa vivacité en maintenant la confrontation avec le monde contemporain.

La transmission de la psychanalyse est loin de constituer une simple passation de concepts; c'est plutôt un processus de transformation interne mettant en jeu la filiation, le transfert, toutes sortes d'identification transgénérationnelles à des personnes et des théories. Cela requiert un dispositif spécial pour la transmission, avec une remise en cause des enseignants par eux-mêmes et par les autres, et un regard sur ce qui ne se transmet pas malgré la volonté de le faire. La psychanalyse est une source intarissable de 
découvertes si l'intégration de la pensée freudienne a permis deuils et dépassements des imitations factices.

Le travail avec les bébés et leurs parents confronte encore le thérapeute aux émois primitifs et aux traumas précoces. La violence des identifications projectives de patients immatures, de nourrissons, de malades, de certains parents, demande un travail qui consiste à contenir un contre-transfert difficile. C'est en supervision que ce travail d'élaboration peut se faire, permettant alors de pouvoir réinterroger les formulations freudiennes. Peut alors se constituer ce que Bolognini appelle le fantasme d'une famille analytique, introjection de nos objets analytiques et identification aux superviseurs, enseignants, à l'ambiance de l'institut et à la relation que chacun entretient avec les auteurs de la psychanalyse.

\section{Le Parent-Infant Program}

Pendant 2 ans, un groupe d'élèves se réunit deux fois par semaine, un soir pour un séminaire de lecture et discussion de textes sur la vie psychique dans le développement de l'enfant et la découverte de la parentalité, et un autre soir pour le groupe de supervision - suivant l'approche de la Tavistock Clinic -, qui effectue des observations à domicile d'un bébé depuis la naissance jusqu'à l'anniversaire de ses 2 ans. Chaque étudiant fait un stage dans une institution qui reçoit des mères en détresse avec leurs jeunes enfants, participe aux groupes de parents et prend en thérapie une dyade, travail supervisé chaque semaine par un des enseignants du Parent-infant program. Un travail de documentation écrit doit être rendu chaque année: il doit d'abord décrire la vie psychique d'un enfant durant sa première année de vie et ensuite rendre compte des cas pris en traitement.

La formation s'adresse aux jeunes et moins jeunes, mais aussi aux collègues qui traitent des adultes, offrant un éclairage sur l'archaïque et le profond des temps précoces, avec l'accès à des populations défavorisées dont la parentalité comme l'enfance sont en danger.

C'est à l'aide de vignettes cliniques et de références théoriques classiques et contemporaines, de L'esquisse pour une psychologie scientifique de 1895 jusqu'aux travaux post-kleiniens et post-Bion, en passant bien sûr par Winnicott, Green et Anzieu, que nous enseignons les débuts de la vie psychique et la rigueur de la pratique analytique.

Les éléments fondamentaux de cet enseignement sont:

- La théorie des pulsions, essentielle chez Freud, mais contestée aux États-Unis, qui affirme le rôle essentiel de la libido dans la formation 
et le maintien de la vie psychique. La sensualité du corps de l'enfant investie par la mère en écho à son propre corps sensoriel et excitable est la source de la sexualité infantile, élément constructeur des instances psychiques.

- La théorie économique, pour laquelle la quantité d'excitation est régulée par le principe de plaisir et l'organisation psychosomatique. La fonction de contenant de la mère est ici au centre. Le Moi Peau et les enveloppes jouent ici un rôle majeur pour le narcissisme primaire.

- L'effet du négatif et de la destructivité lorque ces deux fonctions sont mal régulées, comportant alors un danger immédiat pour le développement. Les réactions de retrait, de détachement et de désinvestissement chez le bébé comme chez la mère sont à repérer le plus tôt possible, puisque, dans le cas contraire, il devient difficile de mobiliser des ressources libidinales suffisantes et les réactions masochistes se développent rapidement.

- Le rôle majeur des traumatismes précoces et des transmissions intergénérationnelles. La plupart sont réparables par la dyade, avec l'aide de l'analyste. Le traumatisme est repéré comme un évènement dont l'enfant serait non pas une victime passive, mais un partenaire actif, malgré son immaturité.

- La spécificité de la vie psychique des parents, confrontés à un processus de deuil de leurs imagos infantiles idéalisées, qui les précipitent parfois dans des défenses narcissiques et des projections incompatibles avec le self en développement de l'enfant.

- Le rôle majeur des fonctionnements transitionnels, tant chez les parents que chez les tout-petits. Les rythmes réguliers de présence et d'absence, associés à l'auto-érotisme, offrent à l'enfant la possibilité d'être seul avec lui-même et de rêver la présence de l'objet. Les précurseurs des jeux transitionnels sont importants à repérer et à favoriser. De même, la thérapie sera fondée sur la capacité ludique de l'analyste et son utilisation des métaphores autant que son langage non verbal. La technique spécifique à ces traitements permet en effet de s'adresser parfois directement au bébé, éveillant chez lui le sens d'un soutien narcissique et communiquant aux parents des messages essentiels sur sa vie interne.

- Les notions de transfert et de contre-transfert comme moteurs des thérapies, avec l'apport de la notion d'identification projective. En 
ce sens, le travail psychothérapeutique est à voir comme un travail de représentation et de figurabilité.

- Enfin, le rôle formateur de l'observation d'un nourrisson à son domicile.

La régularité hebdomadaire de l'observation, l'intensité de l'attention portée au bébé et à son environnement, l'impact des projections et enfin les mouvements inconscients et émotionnels suscités par la présence d'un nourrisson sans défense mais pulsionnel, mettent l'observateur en situation d'extrême sensibilité aux émois primitifs et à ses propres projections inconscientes. Le groupe de supervision sert à contenir les affects et à les transformer en pensée et à favoriser la prise de conscience, à travers le groupe, du mouvement d'auto-analyse ainsi mis en route.

La supervision individuelle permet aussi ce mouvement de prise de conscience, d'introspection et de connaissance. Les étudiants deviennent capables eux aussi de développer l'équivalent d'une préoccupation maternelle, faite d'empathie et d'ambivalence, ainsi que d'une disponibilité à accueillir et savoir lire les enjeux actifs dans une dyade mère-bébé.

Le programme se complète par des séances de cours portant sur les recherches récentes en neuroscience, sur la psychopathologie de la petite enfance et de la parentalité et enfin sur les techniques d'intervention, que ce soit en institution ou dans des thérapies analytiques.

\section{Psychanalyse avec des bébés}

Ce que montre le travail psychanalytique avec des nourrissons, c'est combien l'expérience sensorielle est essentielle à l'instauration du Moi, pour qui les qualités de plaisir et de déplaisir avec le corps, l'oralité, la digestion, le toucher, la solidité musculaire seront autant de marqueurs de la relation avec l'objet. Les troubles significatifs de l'alimentation, du sommeil, de la posture du corps et de l'équilibre psychosomatique de la petite enfance sont associés avec des désinvestissements parfois dommageables pour l'appareil psychique et ses capacités de représentation, ce qui se manifestera ultérieurement à travers des capacités de symbolisation et de jeu très handicapées. Les interventions psychanalytiques opèrent contre la tendance à détruire le processus représentatif, tendance qui consiste en des attaques contre les liens comme dit Bion et qui est ainsi un potentiel désorganisateur associé au clivage du Moi, «une déchirure dans le Moi qui ne guérira jamais plus» (Freud).

En 1967, Donald Winnicott fait paraitre un article étonnant en allemand à propos d'un bébé de 4 mois et demi qui refusait activement le sein 
maternel, état critique qui avait commencé à l'âge de 2 mois, moment auquel l'enfant avait été mis en présence du père.

Winnicott fait l'hypothèse d'une influence de l'angoisse maternelle et de conflits propres à la mère à l'égard de la féminité. Il ajoute l'existence d'un élément, précocement développé, du Surmoi de l'enfant.

Cela le conduit à faire une intervention inhabituelle: il met sa main entre le sein de la mère et la bouche de l'enfant, de façon à déplacer le mouvement agressif du bébé. Il observe alors avec une grande attention combien le bébé se précipite à nouveau sur le mamelon pour se nourrir, et se réorganise.

Winnicott poursuit son intervention en observant l'oralité du nourrisson et l'usage auto-érotique de ses doigts, et il en déduit une relation entre l'agressivité orale et la destructivité aux débuts de la vie. Les deux sensations, orale et tactile, stimulées en même temps par le fait de sucer les doigts, manifestent le rôle de la surface corporelle et de la peau au service des pulsions partielles orales et du plaisir auto-érotique. La capacité de calmer les tensions par une sensation tactile associée à la bouche serait une des premières réalisations du Moi primitif selon Willy Hoffer (1950), que cite d'ailleurs abondamment Winnicott: il en irait d'un Moi primitif déjà différencié du Ça.

Plus spécifiquement, Winnicott cite Willy Hoffer dans ses observations de nourrisson et ses écrits sur l'importance de la libidinisation du corps comme protection contre l'autodestructivité, qui expliquerait l'intensité de l'agressivité tournée vers le monde externe, suivie d'une croissance du narcissisme avec le sentiment de contrôle qui y est associé. Le narcissisme infantile est ici considéré surtout comme une protection contre l'autodestructivité et non contre des dangers externes.

Dans le travail analytique avec les tout-petits, il est important d'associer à la fois la compréhension du fonctionnement inconscient maternel et celle des angoisses primaires du bébé avec les défenses primitives et le besoin de contenance; l'analyste fait des interventions et des interprétations qui aident l'enfant à diminuer la tension anxieuse et à retrouver un plaisir libidinal.

La grande fragilité des débuts de la vie rend essentiel «le rôle de la réciprocité dans la relation, l'expérience d'être porté, tenu, vu et rencontré dans un geste créatif» (Winnicott, 1970). L'intimité si précieuse peut être détruite par un débordement émotionnel non contenu.

Mélanie Klein soulignait pour sa part combien les troubles de la relation avec la mère sont sources d'un développement prématuré et trop strict du Surmoi, avec une influence déterminante sur le développement du sujet. 
Il parait important alors de porter attention à la libidinisation du corps, du Moi-corporel, qui protégera le tout-petit des conséquences d'expériences traumatiques ayant déclenché angoisse, accumulation de rage et attaques associées à l'autodestructivité.

L'apparition précoce d'un Surmoi découle aussi de l'incapacité maternelle à recevoir et à transformer cette angoisse et cette destructivité que l'enfant ne peut que décharger. Une des conséquences de cette incapacité est le fantasme d'objet persécuteur, qui exercera un rôle de Surmoi destructeur et rendra impossible l'accès à la position dépressive - avec le risque d'arrêt du développement et ses troubles graves associés. Bion affirme ainsi, dans Attaques contre les liens: "Le lien entre le nourrisson et le sein dépend de l'identification projective et d'une capacité d'introjecter les identifications projectives. L'impossibilité d'introjecter fait que l'objet extérieur apparaît intrinsèquement hostile à la curiosité et à la méthode - c'est-à-dire l'identification projective par laquelle le nourrisson cherche à la satisfaire. Quand bien même le sein serait ressenti comme fondamentalement compréhensif, il a été transformé par l'envie et la haine du nourrisson en un objet dont l'avidité dévorante a pour but l'introjection des identifications projectives du nourrisson afin de les détruire. C'est ce qu'on voit dans le cas où le patient croit que l'analyste, en comprenant le patient, cherche à le rendre fou. Le résultat est un objet qui, lorsqu'il s'installe chez le patient, joue le rôle d'un Surmoi sévère et destructeur du Moi.» (Bion, 1959, p. 296)

Bion met ici l'accent sur la destructivité dirigée spécifiquement contre la capacité d'entrer en relation avec l'objet maternel; l'agressivité n'est pas seulement intrapsychique, mais aussi interpersonnelle, comme on peut l'observer dans les réactions psychotiques maternelles du post-partum, quand les projections sur le nouveau-né sont hautement agressives. Chez Bion, la pulsion de mort se manifeste avec la peur d'être anéanti: c'est cette agonie primitive que la mère reçoit de son nourrisson, contient et transforme en un message qui vient d'une véritable mise en sens de l'expérience de décharge émotionnelle.

Supporter et absorber cette expérience d'agonie présente un risque de désorganisation pour certains parents. La capacité maternelle d'imaginer joue ici un rôle essentiel et, si elle prend la forme de projections psychotiques, c'est le pouvoir de symbolisation de l'enfant qui risque d'être endommagé. Le rôle du parent d'un tout-petit est d'être «le penseur qui pense les pensées», écrit Grotstein en 1981, «le rêveur qui rêve le rêve»: il s'agit d'offrir une réceptivité qui permet d'associer le familier avec ces expériences 
si étranges. "L'alchimie digestive de transformation» requiert ce processus créatif fondé sur l'imagination; ce n'est donc pas une capacité intellectuelle, bien que ce soit la source de la capacité de penser.

Le psychanalyste qui travaille avec les bébés et leurs parents intervient directement avec l'enfant, faisant preuve de cette faculté d'imagination, de transformation et de compréhension des défenses spécifiques du Moi primitif.

Si l'on fait l'hypothèse que le nourrisson débordé de tensions anxieuses est en quête d'apaisement, la communication de l'analyste avec l'enfant peut être un facteur essentiel de changement: il s'agit d'intervenir pour libérer les affects du bébé qui sont censés être à l’origine du symptôme. En même temps, l'échange entre l'analyste et l'enfant donne à la mère ou aux parents présents une possibilité de sens, de compréhension des troubles dans la dyade et de leur part dans cette dynamique. L'attention la plus calme possible de l'analyste, qui se manifeste dans sa façon de s'adresser à l'enfant, permet souvent à la mère de se détendre elle aussi et d'être plus attentive aux signaux de son nourrisson.

La métapsychologie freudienne est un outil majeur des thérapies analytiques. Depuis les travaux de pionniers comme Willy Hoffer et Winnicott, l'intérêt pour les notions de Moi corporel, d'investissement libidinal à l'origine de la vie psychique, de transfert - même avec les tout-petits -, de Surmoi primitif et de manifestations masochistes chez des bébés démunis est devenu majeur pour traiter les tout-petits sérieusement, même si cela reste surprenant pour certains de nos collègues.

\section{Pour conclure}

Transmettre connaissances et pratiques analytiques est essentiel, mais il faut surtout pouvoir former les plus jeunes à trouver par eux-mêmes de nouveaux moyens de penser et d'intervenir dans le monde moderne, tout en leur disant la souffrance de l'analyste devant les situations d'impasse et les cas difficiles. La violence familiale et sociale, des immigrants aux couples fous, aux dyades abusives, montrent le rôle majeur des politiques de santé mentale. Le souci de pouvoir atteindre les patients, surtout ceux en situation de précarité, est à transmettre dans tout travail de formation.

Dans le monde contemporain, les bébés sont élevés en ayant la technologie comme jeu transitionnel ou obsessionnel chez leurs parents et, parfois, en ayant le téléphone portable ou la tablette comme baby-sitter. Certains ont voyagé dans la détresse avec leurs parents refugiés en quête d'asile, déjà 
trimbalés dans le chaos avec des parents traumatisés. La dépression qui s’accroit dans le monde a été nommée maladie mondiale majeure par l'Organisation mondiale de la santé, que ce soit la dépression du post-partum ou la dépression primaire du tout-petit qui se manifeste à travers la gamme des maladies psychosomatiques. Éthique et responsabilité font partie de ce travail.

Mais le plaisir du travail analytique et celui de se confronter à de jeunes esprits avides de connaitre et comprendre rendent l'enseignement et la transmission une éternelle source de jouvence.

\section{Christine Anzieu-Premmereur canzieu@gmail.com}

\section{Références}

Anzieu-Premmereur, C. (2011). Fondements maternels de la vie psychique. Revue française de psychanalyse, 75 (5), 1449-1488.

Bick, E. (1968). L'expérience de la peau dans les relations objectales précoces. Revue belge de psychanalyse, 50 (2007), 137-141.

Bion, W. (1962). Aux sources de l'expérience. Paris: Presses universitaires de France.

Bowlby, J. (2002). Attachement et perte: Vol. 1. L'attachement. Paris: Presses universitaires de France.

Bullinger, A. (1998). Le dialogue sensorimoteur avec l'enfant: les particularités du bébé à risque autistique. Dans P. Delion (dir.), Les bébés à risque autistique (p. 47-60). Paris: Eres.

Donnet, J.-L. (1975). Le divan bien tempéré. Nouvelle revue de psychanalyse, 8, 23-49.

Cahn, R. (2002). La fin du divan? Paris: Odile Jacob.

Fraiberg, S. (1980). Fantômes dans la chambre d'enfants. Paris: Presses universitaires de France.

Freud, S. (1915). Pulsions et destin des pulsions. Dans S. Freud (1976), Métapsychologie (p. 11-44). Paris: Gallimard.

Freud, S. (1923). Le Moi et le Ça. Dans S. Freud (1991), Euvres complètes. Vol. XVI (p. 255301). Paris: Presses universitaires de France.

Hoffer, W. (1950). Mouth, hand and ego integration. Psychoanalytic Study of the Child, 3-4, 49-56.

Klein, M. (1952). Quelques conclusions théoriques au sujet de la vie émotionnelle des bébés. Dans M. Klein et al. (1980), Développements de la psychanalyse (p. 187-222). Paris: Presses universitaires de France.

Green, A. (1983). La mère morte. Dans A. Green, Narcissisme de vie, narcissisme de mort (p. 222-254). Paris: Éditions de Minuit.

Grotstein, J. S. (1981). La «transidentification projective» : une extension du concept d'identification projective, Année psychanalytique internationale, 4 (2006), 121-139.

M'Uzan, M. de (2008). La chimère des inconscients. Paris: Presses universitaires de France.

Milner, M. (1976). L'inconscient et la peinture. Paris: Presses universitaires de France.

Parat, C. (1995). L'affect partagé. Paris: Presses universitaires de France.

Roussillon, R. (2008). Le transitionnel, le sexuel et la réflexivité. Paris: Dunod. 
Schacht, L. (1998). À propos de la signification de la confiance en analyse d'enfant. Journal de la psychanalyse de l'enfant, 22, 39-63.

Searles, H. (1981). Le contre-transfert. Paris: Gallimard.

Winnicott, D. W. (1941). L'observation des jeunes enfants dans une situation établie. Dans D. W. Winnicott (1989, 2 éd.), De la pédiatrie à la psychanalyse (p. 269-288). Paris: Payot.

Winnicott, D. W. (1947a). Le bébé en tant que personne. Dans D. W. Winnicott (1982), L'enfant et le monde extérieur (p. 103-112). Paris: Payot.

Winnicott, D. W. (1947 b). La haine dans le contre-transfert. Dans D. W. Winnicott (1989, $2^{\mathrm{e}}$ éd.), De la pédiatrie à la psychanalyse (p. 48-58). Paris: Payot.

Winnicott, D. W. (1951). Objets transitionnels et phénomènes transitionnels. Dans D. W. Winnicott (1989, $2^{\mathrm{e}}$ éd.), De la pédiatrie à la psychanalyse (p. 109-125). Paris: Payot.

Winnicott, D. W. (1967). Eine Kinderbeobachtung. Psyche, 21 (10-11), 848-852.

Winnicott, D. W. (1968). L'utilisation de l'objet et le mode de relation à l'objet au travers des identifications. Dans D. W. Winnicott (1971), Jeu et réalité (p. 120-130). Paris: Gallimard. 